\title{
On-site Renewable Energy and Green Buildings: A System-Level Analysis
}

\author{
Sami G. Al-Ghamdi and Melissa M. Bilec ${ }^{1}$
}

Civil and Environmental Engineering, University of Pittsburgh, Pittsburgh, PA 15261, USA

\section{SUPPLEMENTARY INFORMATION (A)}

The following is a supplementary information for the 25 sites included in the study.

\section{List of supplementary figures}

Figure S1. Distribution of the 25 locations within the study by power plant type/energy sources used. .1

\section{List of supplementary tables}

Table S1. Development of renewable energy requirements in different LEED versions ..........................................2

Table S2. Electric power plant sources details in the 25 locations in the study site ..................................................

Table S3. Annual electricity requirements and coast in the 25 locations in the study site ........................................

Table S4. Annual on-site electricity production from PV and wind-turbines in all locations .....................................5

Table S5. Summary of on-site production contribution to the building's electricity requirements .............................6

Table S6. Summary of photovoltaic analysis; installed panel area and cost; payback period ....................................

Table S7. Annual Life Cycle $\mathrm{CO}_{2}$ equivalent emissions in the 25 locations included in the study ............................8

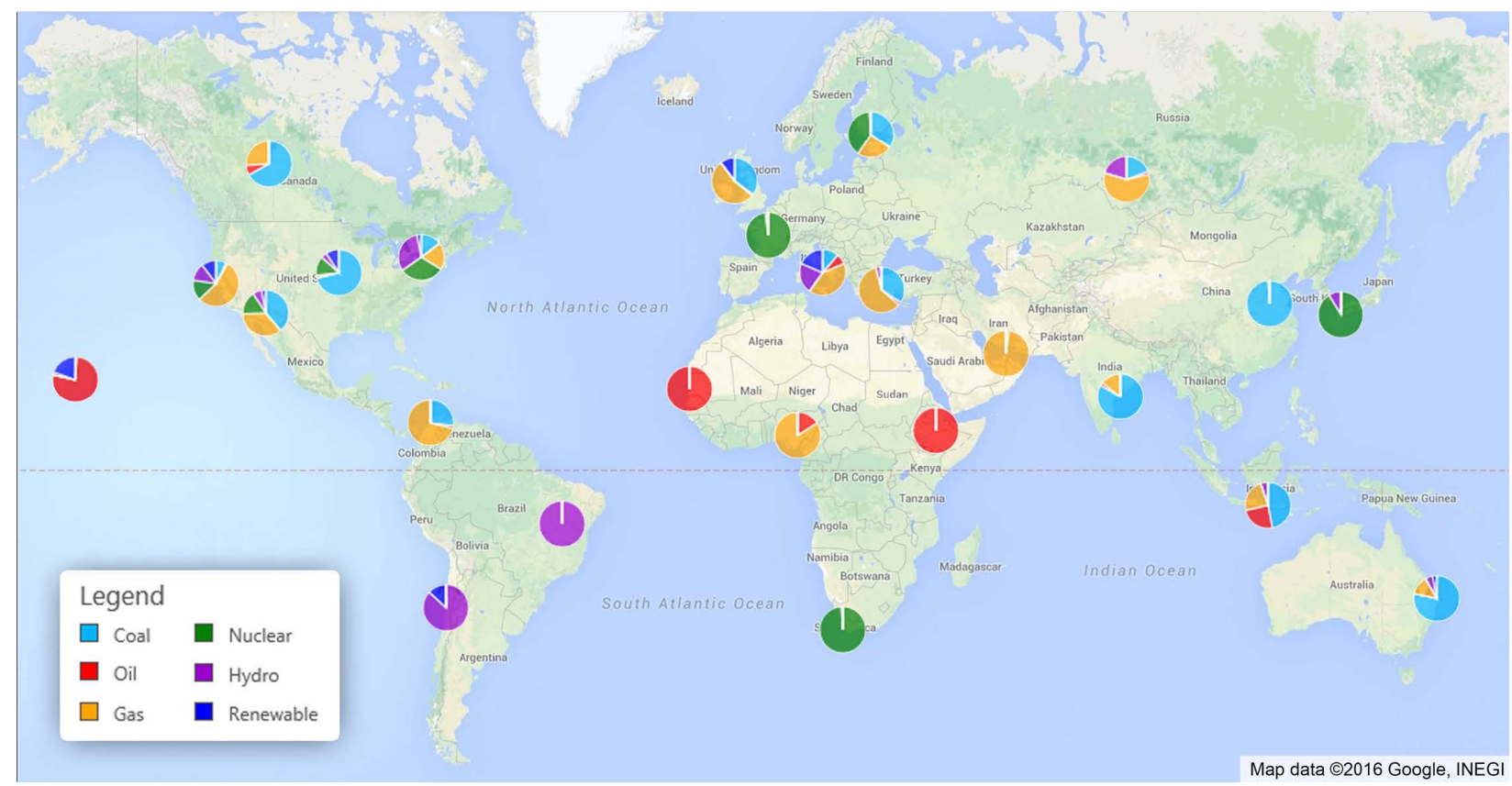

Figure S1. Distribution of the 25 locations within the study by power plant type/energy sources used

\footnotetext{
${ }^{1}$ Corresponding author: Tel.: +1 (412) 648-8075; Fax: +1 (412) 624-0135. E-mail address: mbilec@pitt.edu
} 
Table S1. Development of renewable energy requirements in different LEED versions

\begin{tabular}{|c|c|c|c|c|c|c|}
\hline Version & Credit & Title & Requirements & Points & Point's \%* & Summary of changes and notes \\
\hline $\begin{array}{l}\text { LEED v2.0, } \\
2001\end{array}$ & $\begin{array}{l}\text { EAc2.1 } \\
\text { EAc2.2 } \\
\text { EAc2.3 }\end{array}$ & $\begin{array}{l}\text { Renewable } \\
\text { Energy }\end{array}$ & $\begin{array}{c}0 \% \text { for } \\
2-Y \text { Contract }\end{array}$ & $\begin{array}{l}1 \\
2 \\
3\end{array}$ & $\begin{array}{c}6 \% \text { of } \\
\text { EA points }\end{array}$ & $\begin{array}{l}\text { - Energy referenced standard start with ASHRAE } \\
90.1-1999, \text { the total EA points represent } 25 \% \text { of the } \\
\text { total points in LEED v2.0 } \\
\text { - Credit EAc2 first released as "Renewable Energy" } \\
\text { with three possible points. } \\
\text { - Credit EAc6 first released as "Green Power" with } \\
\text { one possible point. }\end{array}$ \\
\hline $\begin{array}{l}\text { LEED v2.1, } \\
\quad 2003\end{array}$ & $\begin{array}{c}\text { EAc2.1 } \\
\text { EAc2.2 } \\
\text { EAc2.3 } \\
\text { EAc6 }\end{array}$ & $\begin{array}{l}\text { Renewable } \\
\text { Energy }\end{array}$ & $\begin{array}{c}5 \% \\
10 \% \\
20 \% \\
\\
50 \% \text { for } \\
2-Y \text { Contract }\end{array}$ & $\begin{array}{l}1 \\
2 \\
3\end{array}$ & $\begin{array}{l}18 \% \text { of } \\
\text { EA points } \\
\quad 6 \% \text { of } \\
\text { EA points }\end{array}$ & $\begin{array}{l}\text { Energy referenced standard remain as ASHRAE } \\
90.1-1999, \text { the total EA points represent } 25 \% \text { of the } \\
\text { total points in LEED v2.1. } \\
\text { Overall no substantive changes, except for } \\
\text { defining the required percentage for green power of } \\
50 \% \text {. }\end{array}$ \\
\hline $\begin{array}{l}\text { LEED v2.2, } \\
\quad 2005\end{array}$ & $\begin{array}{l}\text { EAc2.1 } \\
\text { EAc2.2 } \\
\text { EAc2.3 } \\
\text { EAc6 }\end{array}$ & $\begin{array}{l}\text { On-Site } \\
\text { Renewable } \\
\text { energy } \\
\text { Green Power }\end{array}$ & $\begin{array}{c}2.5 \% \\
7.5 \% \\
12.5 \% \\
\\
35 \% \text { for } \\
2 \text {-Y Contract }\end{array}$ & $\begin{array}{l}1 \\
2 \\
3\end{array}$ & $\begin{array}{c}6 \% \text { of } \\
\text { EA points }\end{array}$ & $\begin{array}{l}\text { - Energy referenced standard updated to ASHRAE } \\
90.1-2004, \text { the total EA points represent } 25 \% \text { of the } \\
\text { total points in LEED v } 2.2 \\
\text { - Credit EAc2 title renamed from "Renewable } \\
\text { Energy" } \\
\text { - Credit Eac6, percentage reduced from } 50 \% \text { to } \\
35 \% \text {. }\end{array}$ \\
\hline $\begin{array}{l}\text { LEED v3.0, } \\
2009\end{array}$ & $\begin{array}{c}\text { EAc2.1 } \\
\text { EAc2.2 } \\
\text { EAc2.3 } \\
\text { EAc2.4 } \\
\text { EAc2.5 } \\
\text { EAc2.6 } \\
\text { EAc2.7 } \\
\text { EAc6 }\end{array}$ & $\begin{array}{l}\text { On-Site } \\
\text { Renewable } \\
\text { Energy }\end{array}$ & $\begin{array}{c}1 \% \\
3 \% \\
5 \% \\
7 \% \\
9 \% \\
11 \% \\
13 \%\end{array}$ & $\begin{array}{l}1 \\
2 \\
3 \\
4 \\
5 \\
6 \\
7\end{array}$ & $\begin{array}{c}6 \% \text { of } \\
\text { EA points }\end{array}$ & $\begin{array}{l}\text { - Energy referenced standard updated to ASHRAE } \\
90.1-2007 \text {, the total EA points represent } 32 \% \text { of the } \\
\text { total points in LEED v3.0. } \\
\text { - Credit EAc2 points reweighted from } 1-3 \text { points to } \\
1-7 \text { points, nevertheless the share of EA points } \\
\text { remains slightly unchanged, because the entire EA } \\
\text { section has been increased from } 25 \% \text { to } 32 \% \text {. Also, } \\
\text { Lower and higher thresholds added } \\
\text { - Credit EAc6, reweighted from } 1 \text { point to } 2 \text { points } \\
\text { and purchases of green power are based on the } \\
\text { quantity of energy consumed, not cost. Also, specify } \\
\text { Green-e Energy products }\end{array}$ \\
\hline $\begin{array}{l}\text { LEED v4.0, } \\
\qquad 2013\end{array}$ & $\begin{array}{l}\text { EAc5.1 } \\
\text { EAc5.2 } \\
\text { EAc5.3 }\end{array}$ & $\begin{array}{c}\text { Green Power } \\
\text { and Carbon } \\
\text { Offsets }\end{array}$ & $\begin{array}{l}50 \% \text { for } \\
5-Y \text { Contract } \\
100 \% \text { for } \\
5 \text {-Y Contract }\end{array}$ & $\begin{array}{l}1 \\
2 \\
3\end{array}$ & $\begin{array}{c}6 \% \text { of } \\
\text { EA points }\end{array}$ & $\begin{array}{l}\text { Energy referenced standard updated to ASHRAE } \\
90.1-2010 \text {, the total EA points represent } 30 \% \text { of the } \\
\text { total points in LEED v4.0 } \\
\text { - Credit EAc2 title renamed from "On-Site } \\
\text { Renewable Energy" and points adjusted } \\
\text { significantly. Also, provision for community-scale } \\
\text { renewable energy systems was added. EAc } 2.2 \text { is not } \\
\text { applicable to NC rating system. } \\
\text { - Credit EAc6 title renamed from "Green Power". } \\
\text { The required percentage has been increased. Credit } \\
\text { based on total building energy usage. Carbon offsets } \\
\text { allowed for scope } 1 \text { or } 2 \text { emissions. Required } \\
\text { contract length extended from } 2 \text { years to } 5 \text { years. } \\
\text { Eligible resources must have come online after } \\
\text { January } 1,2005 \text {. } \\
\text { - New pilot credit (1 point) titled "Renewable } \\
\text { energy - distributed generation", to make the } \\
\text { building structure capable of supporting planned } \\
\text { photovoltaic technologies on the roof (Solar facility } \\
\text { capacity: } 250,500 \text { or } 1,000 \mathrm{~kW} \text { ). }\end{array}$ \\
\hline
\end{tabular}

* Point's \%: represents the proportion of renewable energy or green power points out of the total points in the Energy and Atmosphere (EA) category. 
Table S2. Electric power plant sources details in the 25 locations in the study site*

\begin{tabular}{|c|c|c|c|c|c|c|c|c|}
\hline ID & Locations Info & Coal & Oil & Gas & Nuclear & Hydro & Renewable & Other \\
\hline Nat'l- 098 & Sodus, New York, United States & $15 \%$ & $1 \%$ & $19 \%$ & $31 \%$ & $31 \%$ & $4 \%$ & $0 \%$ \\
\hline Nat'l- 077 & Honokaa, Hawaii, United States & $2 \%$ & $77 \%$ & $0 \%$ & $0 \%$ & $2 \%$ & $19 \%$ & $0 \%$ \\
\hline Nat'1-035 & Farragut, Iowa, United States & $69 \%$ & $0 \%$ & $2 \%$ & $14 \%$ & $4 \%$ & $10 \%$ & $0 \%$ \\
\hline Nat'1-099 & Phoenix, Arizona, United States & $39 \%$ & $0 \%$ & $36 \%$ & $17 \%$ & $6 \%$ & $3 \%$ & $0 \%$ \\
\hline Nat'1-075 & Annapolis, California, United States & $7 \%$ & $1 \%$ & $53 \%$ & $15 \%$ & $13 \%$ & $10 \%$ & $1 \%$ \\
\hline Int'1-056 & Fort McMurray, Alberta, Canada & $67 \%$ & $7 \%$ & $26 \%$ & $0 \%$ & $0 \%$ & $0 \%$ & $0 \%$ \\
\hline Int'l-106 & Vantaa, Southern Finland, Finland & $33 \%$ & $1 \%$ & $24 \%$ & $39 \%$ & $2 \%$ & $1 \%$ & $0 \%$ \\
\hline Int'1-037 & Barreiras, Bahia, Brazil & $0 \%$ & $0 \%$ & $0 \%$ & $0 \%$ & $100 \%$ & $0 \%$ & $0 \%$ \\
\hline Int'1-280 & Söke, Aydın, Turkey & $34 \%$ & $3 \%$ & $58 \%$ & $0 \%$ & $4 \%$ & $2 \%$ & $0 \%$ \\
\hline Int'1-151 & Banjar, Bali, Indonesia & $46 \%$ & $25 \%$ & $24 \%$ & $0 \%$ & $5 \%$ & $0 \%$ & $0 \%$ \\
\hline Int'l-214 & Makurdi, Benue, Nigeria & $0 \%$ & $16 \%$ & $84 \%$ & $0 \%$ & $0 \%$ & $0 \%$ & $0 \%$ \\
\hline Int'l-289 & Belfast, United Kingdom & $35 \%$ & $2 \%$ & $53 \%$ & $0 \%$ & $0 \%$ & $10 \%$ & $0 \%$ \\
\hline Int'l-192 & Nouakchott, Mauritania & $0 \%$ & $100 \%$ & $0 \%$ & $0 \%$ & $0 \%$ & $0 \%$ & $0 \%$ \\
\hline Int'l-014 & Gold Coast, Queensland, Australia & $76 \%$ & $1 \%$ & $14 \%$ & $0 \%$ & $5 \%$ & $4 \%$ & $0 \%$ \\
\hline Int'l-256 & Stellenbosch, South Africa & $0 \%$ & $0 \%$ & $0 \%$ & $99 \%$ & $1 \%$ & $0 \%$ & $0 \%$ \\
\hline Int'l-284 & Ajman, United Arab Emirates & $0 \%$ & $2 \%$ & $98 \%$ & $0 \%$ & $0 \%$ & $0 \%$ & $0 \%$ \\
\hline Int'1-230 & Berdsk, Novosibirskaya, Russia & $20 \%$ & $2 \%$ & $57 \%$ & $0 \%$ & $20 \%$ & $0 \%$ & $0 \%$ \\
\hline Int'1-064 & Heze, Shandong, China & $99 \%$ & $1 \%$ & $0 \%$ & $0 \%$ & $0 \%$ & $0 \%$ & $0 \%$ \\
\hline Int'1-159 & Naples, Campania, Italy & $12 \%$ & $7 \%$ & $40 \%$ & $0 \%$ & $22 \%$ & $19 \%$ & $0 \%$ \\
\hline Int'l-063 & Coquimbo, Elqui, Chile & $0 \%$ & $0 \%$ & $0 \%$ & $0 \%$ & $86 \%$ & $14 \%$ & $0 \%$ \\
\hline Int'l-139 & Narasapur, India & $82 \%$ & $3 \%$ & $15 \%$ & $0 \%$ & $0 \%$ & $0 \%$ & $0 \%$ \\
\hline Int'l-103 & Dessie, Amhara, Ethiopia & $0 \%$ & $100 \%$ & $0 \%$ & $0 \%$ & $0 \%$ & $0 \%$ & $0 \%$ \\
\hline Int'l-113 & Châteauroux, Centre, France & $1 \%$ & $0 \%$ & $1 \%$ & $95 \%$ & $3 \%$ & $1 \%$ & $0 \%$ \\
\hline Int'l-167 & Ōita, Japan & $23 \%$ & $7 \%$ & $23 \%$ & $42 \%$ & $5 \%$ & $0 \%$ & $0 \%$ \\
\hline Int'l-084 & Sabanalarga, Atlántico, Colombia & $27 \%$ & $2 \%$ & $71 \%$ & $0 \%$ & $0 \%$ & $0 \%$ & $0 \%$ \\
\hline
\end{tabular}

* The data in this table are represented in Figure S1 in this document (SI-A) 
Table S3. Annual electricity requirements and coast in the 25 locations in the study site*

\begin{tabular}{|c|c|c|c|c|}
\hline $\begin{array}{l}\text { Project } \\
\text { ID }\end{array}$ & $\begin{array}{l}\text { Locations } \\
\text { Info }\end{array}$ & $\begin{array}{c}\text { ASHREA } \\
\text { Climate Zone }\end{array}$ & $\begin{array}{c}\text { Annual Electricity } \\
\text { Requirements (MWh) }\end{array}$ & $\begin{array}{c}\text { Annual Electricity Costs } \\
\text { from Grid (\$) }\end{array}$ \\
\hline Nat'l- 098 & Sodus, New York, United States & $5 \mathrm{~A}$ & 540 & $\$ 75,644$ \\
\hline Nat'l- 077 & Honokaa, Hawaii, United States & $2 \mathrm{~A}$ & 628 & $\$ 213,688$ \\
\hline Nat'l-035 & Farragut, Iowa, United States & $5 \mathrm{~A}$ & 596 & $\$ 41,748$ \\
\hline Nat'l-099 & Phoenix, Arizona, United States & $2 \mathrm{~B}$ & 699 & $\$ 62,930$ \\
\hline Nat'l-075 & Annapolis, California, United States & $3 \mathrm{C}$ & 554 & $\$ 66,456$ \\
\hline Int'l-056 & Fort McMurray, Alberta, Canada & 7 & 556 & $\$ 55,623$ \\
\hline Int'l-106 & Vantaa, Southern Finland, Finland & $6 \mathrm{~A}$ & 548 & $\$ 54,780$ \\
\hline Int'l-037 & Barreiras, Bahia, Brazil & $1 \mathrm{~A}$ & 501 & $\$ 75,141$ \\
\hline Int'l-280 & Söke, Aydın, Turkey & $3 \mathrm{~A}$ & 625 & $\$ 87,437$ \\
\hline Int'l-151 & Banjar, Bali, Indonesia & $2 \mathrm{~A}$ & 631 & $\$ 37,877$ \\
\hline Int'l-214 & Makurdi, Benue, Nigeria & $1 \mathrm{~A}$ & 789 & $\$ 71,030$ \\
\hline Int'1-289 & Belfast, United Kingdom & $5 \mathrm{~A}$ & 501 & $\$ 75,141$ \\
\hline Int'l-192 & Nouakchott, Mauritania & 1B & 734 & $\$ 66,084$ \\
\hline Int'1-014 & Gold Coast, Queensland, Australia & $2 \mathrm{~A}$ & 645 & $\$ 38,709$ \\
\hline Int'1-256 & Stellenbosch, South Africa & $3 \mathrm{C}$ & 572 & $\$ 11,450$ \\
\hline Int'l-284 & Ajman, United Arab Emirates & 1B & 820 & $\$ 73,806$ \\
\hline Int'l-230 & Berdsk, Novosibirskaya, Russia & 7 & 553 & $\$ 27,659$ \\
\hline Int'1-064 & Heze, Shandong, China & $3 \mathrm{~A}$ & 640 & $\$ 57,638$ \\
\hline Int'l-159 & Naples, Campania, Italy & $3 \mathrm{~A}$ & 615 & $\$ 172,069$ \\
\hline Int'l-063 & Coquimbo, Elqui, Chile & $3 \mathrm{C}$ & 562 & $\$ 78,666$ \\
\hline Int'1-139 & Narasapur, India & $1 \mathrm{~A}$ & 774 & $\$ 61,940$ \\
\hline Int'l-103 & Dessie, Amhara, Ethiopia & $3 \mathrm{C}$ & 585 & $\$ 52,632$ \\
\hline Int'l-113 & Châteauroux, Centre, France & $4 \mathrm{~A}$ & 565 & $\$ 62,203$ \\
\hline Int'l-167 & Ōita, Japan & $3 \mathrm{C}$ & 597 & $\$ 95,496$ \\
\hline Int'l-084 & Sabanalarga, Atlántico, Colombia & $1 \mathrm{~A}$ & 792 & $\$ 102,911$ \\
\hline
\end{tabular}

* The data in this table are represented in Figure 3 in the paper 
Table S4. Annual on-site electricity production from PV and wind-turbines in all locations*

\begin{tabular}{|c|c|c|c|c|}
\hline $\begin{array}{l}\text { Project } \\
\text { ID }\end{array}$ & $\begin{array}{l}\text { Locations } \\
\text { Info }\end{array}$ & $\begin{array}{c}\text { Annual On-site } \\
\text { Produced from PV } \\
(\mathrm{kWh})\end{array}$ & $\begin{array}{c}\text { Annual On-site } \\
\text { Produced from Wind- } \\
\text { Turbines }(\mathrm{kWh})\end{array}$ & $\begin{array}{l}\text { Annual Saving Costs } \\
\text { from Renewables (\$) }\end{array}$ \\
\hline Nat'l- 098 & Sodus, New York, United States & 222,706 & 46,835 & $\$ 37,736$ \\
\hline Nat'l- 077 & Honokaa, Hawaii, United States & 417,330 & 1,000 & $\$ 142,232$ \\
\hline Nat'l-035 & Farragut, Iowa, United States & - & - & $\$-$ \\
\hline Nat'l-099 & Phoenix, Arizona, United States & 255,068 & 2,235 & $\$ 23,157$ \\
\hline Nat'l-075 & Annapolis, California, United States & 295,196 & 4,520 & $\$ 35,966$ \\
\hline Int'l-056 & Fort McMurray, Alberta, Canada & 107,923 & 2,040 & $\$ 10,996$ \\
\hline Int'l-106 & Vantaa, Southern Finland, Finland & - & - & - \\
\hline Int'l-037 & Barreiras, Bahia, Brazil & 87,292 & 16,615 & $\$ 15,586$ \\
\hline Int'l-280 & Söke, Aydın, Turkey & 333,990 & 7,975 & $\$ 47,875$ \\
\hline Int'l-151 & Banjar, Bali, Indonesia & - & - & - \\
\hline Int'l-214 & Makurdi, Benue, Nigeria & 298,469 & 1,200 & $\$ 26,970$ \\
\hline Int'l-289 & Belfast, United Kingdom & 87,292 & 16,615 & $\$ 15,586$ \\
\hline Int'l-192 & Nouakchott, Mauritania & 324,024 & 21,690 & $\$ 31,114$ \\
\hline Int'l-014 & Gold Coast, Queensland, Australia & 185,575 & 12,025 & $\$ 11,856$ \\
\hline Int'l-256 & Stellenbosch, South Africa & - & - & - \\
\hline Int'l-284 & Ajman, United Arab Emirates & 309,252 & 6,380 & $\$ 28,407$ \\
\hline Int'l-230 & Berdsk, Novosibirskaya, Russia & - & - & - \\
\hline Int'1-064 & Heze, Shandong, China & 182,489 & 3,650 & $\$ 16,753$ \\
\hline Int'l-159 & Naples, Campania, Italy & 335,756 & 5,930 & $\$ 95,672$ \\
\hline Int'1-063 & Coquimbo, Elqui, Chile & 414,174 & 4,350 & $\$ 58,593$ \\
\hline Int'l-139 & Narasapur, India & 175,796 & 4,340 & $\$ 14,411$ \\
\hline Int'l-103 & Dessie, Amhara, Ethiopia & 328,267 & 8,015 & $\$ 30,265$ \\
\hline Int'l-113 & Châteauroux, Centre, France & 134,160 & 8,905 & $\$ 15,737$ \\
\hline Int'l-167 & Ōita, Japan & 263,275 & 3,620 & $\$ 42,703$ \\
\hline Int'l-084 & Sabanalarga, Atlántico, Colombia & 361,276 & 3,235 & $\$ 47,386$ \\
\hline
\end{tabular}

* The data in this table are represented in Figure 3 in the paper 
Table S5. Summary of on-site production contribution to the building's electricity requirements*

\begin{tabular}{|c|c|c|c|}
\hline $\begin{array}{l}\text { Project } \\
\text { ID }\end{array}$ & $\begin{array}{l}\text { Locations } \\
\text { Info }\end{array}$ & $\begin{array}{l}\text { PV production to the building's } \\
\text { requirements }\end{array}$ & $\begin{array}{l}\text { Wind production to the building's } \\
\text { requirements }\end{array}$ \\
\hline Nat'l- 098 & Sodus, New York, United States & $41 \%$ & $9 \%$ \\
\hline Nat'l- 077 & Honokaa, Hawaii, United States & $66 \%$ & $0 \%$ \\
\hline Nat'l-035 & Farragut, Iowa, United States & $0 \%$ & $0 \%$ \\
\hline Nat'1-099 & Phoenix, Arizona, United States & $36 \%$ & $0 \%$ \\
\hline Nat'l-075 & Annapolis, California, United States & $53 \%$ & $1 \%$ \\
\hline Int'1-056 & Fort McMurray, Alberta, Canada & $19 \%$ & $0 \%$ \\
\hline Int'l-106 & Vantaa, Southern Finland, Finland & $0 \%$ & $0 \%$ \\
\hline Int'1-037 & Barreiras, Bahia, Brazil & $17 \%$ & $3 \%$ \\
\hline Int'l-280 & Söke, Aydın, Turkey & $53 \%$ & $1 \%$ \\
\hline Int'l-151 & Banjar, Bali, Indonesia & $0 \%$ & $0 \%$ \\
\hline Int'l-214 & Makurdi, Benue, Nigeria & $38 \%$ & $0 \%$ \\
\hline Int'l-289 & Belfast, United Kingdom & $17 \%$ & $3 \%$ \\
\hline Int'l-192 & Nouakchott, Mauritania & $44 \%$ & $3 \%$ \\
\hline Int'l-014 & Gold Coast, Queensland, Australia & $29 \%$ & $2 \%$ \\
\hline Int'l-256 & Stellenbosch, South Africa & $0 \%$ & $0 \%$ \\
\hline Int'l-284 & Ajman, United Arab Emirates & $38 \%$ & $1 \%$ \\
\hline Int'l-230 & Berdsk, Novosibirskaya, Russia & $0 \%$ & $0 \%$ \\
\hline Int'1-064 & Heze, Shandong, China & $28 \%$ & $1 \%$ \\
\hline Int'l-159 & Naples, Campania, Italy & $55 \%$ & $1 \%$ \\
\hline Int'l-063 & Coquimbo, Elqui, Chile & $74 \%$ & $1 \%$ \\
\hline Int'l-139 & Narasapur, India & $23 \%$ & $1 \%$ \\
\hline Int'l-103 & Dessie, Amhara, Ethiopia & $56 \%$ & $1 \%$ \\
\hline Int'l-113 & Châteauroux, Centre, France & $24 \%$ & $2 \%$ \\
\hline Int'l-167 & Ōita, Japan & $44 \%$ & $1 \%$ \\
\hline Int'l-084 & Sabanalarga, Atlántico, Colombia & $46 \%$ & $0 \%$ \\
\hline
\end{tabular}

* The data in this table are represented in Figure 3 in the paper 
Table S6. Summary of photovoltaic analysis; installed panel area and cost; payback period*

\begin{tabular}{|c|c|c|c|c|}
\hline $\begin{array}{l}\text { Project } \\
\text { ID }\end{array}$ & $\begin{array}{l}\text { Locations } \\
\text { Info }\end{array}$ & $\begin{array}{c}\text { Total PV Installed Panel } \\
\text { Area }\left(\mathrm{ft}^{2}\right)\end{array}$ & $\begin{array}{c}\text { Total PV Installed Panel } \\
\text { Cost (\$) }\end{array}$ & $\begin{array}{l}\text { Total PV Payback } \\
\text { Period (years) }\end{array}$ \\
\hline Nat'l- 098 & Sodus, New York, United States & 18,208 & $\$ 1,868,505$ & 39 \\
\hline Nat'1- 077 & Honokaa, Hawaii, United States & 27,387 & $\$ 3,315,755$ & 19 \\
\hline Nat'l-035 & Farragut, Iowa, United States & - & - & 51 \\
\hline Nat'l-099 & Phoenix, Arizona, United States & 12,778 & $\$ 1,311,278$ & 39 \\
\hline Nat'l-075 & Annapolis, California, United States & 18,081 & $\$ 1,855,472$ & 36 \\
\hline Int'l-056 & Fort McMurray, Alberta, Canada & 8,669 & $\$ 889,613$ & 48 \\
\hline Int'l-106 & Vantaa, Southern Finland, Finland & - & $\$-$ & 56 \\
\hline Int'1-037 & Barreiras, Bahia, Brazil & 19,907 & $\$ 2,042,856$ & 33 \\
\hline Int'1-280 & Söke, Aydın, Turkey & 21,743 & $\$ 2,231,267$ & 34 \\
\hline Int'l-151 & Banjar, Bali, Indonesia & - & - & 61 \\
\hline Int'l-214 & Makurdi, Benue, Nigeria & 15,333 & $\$ 1,573,472$ & 38 \\
\hline Int'1-289 & Belfast, United Kingdom & 9,479 & $\$ 972,735$ & 46 \\
\hline Int'l-192 & Nouakchott, Mauritania & 16,079 & $\$ 1,650,027$ & 37 \\
\hline Int'1-014 & Gold Coast, Queensland, Australia & 8,880 & $\$ 911,266$ & 48 \\
\hline Int'l-256 & Stellenbosch, South Africa & - & - & 87 \\
\hline Int'l-284 & Ajman, United Arab Emirates & 16,012 & $\$ 1,643,151$ & 38 \\
\hline Int'1-230 & Berdsk, Novosibirskaya, Russia & - & - & 80 \\
\hline Int'1-064 & Heze, Shandong, China & 11,063 & $\$ 1,135,285$ & 42 \\
\hline Int'l-159 & Naples, Campania, Italy & 27,564 & $\$ 2,828,618$ & 24 \\
\hline Int'1-063 & Coquimbo, Elqui, Chile & 24,866 & $\$ 2,551,749$ & 31 \\
\hline Int'l-139 & Narasapur, India & 9,446 & $\$ 969,349$ & 43 \\
\hline Int'l-103 & Dessie, Amhara, Ethiopia & 15,520 & $\$ 1,592,662$ & 36 \\
\hline Int'l-113 & Châteauroux, Centre, France & 10,253 & $\$ 1,052,163$ & 45 \\
\hline Int'l-167 & Ōita, Japan & 20,916 & $\$ 2,146,400$ & 36 \\
\hline Int'1-084 & Sabanalarga, Atlántico, Colombia & 21,745 & $\$ 2,231,472$ & 34 \\
\hline
\end{tabular}

* The data in this table are represented in Figures 3 and 4 in the paper 
Table S7. Annual Life Cycle $\mathrm{CO}_{2}$ equivalent emissions in the 25 locations included in the study*

\begin{tabular}{|c|c|c|c|c|}
\hline $\begin{array}{l}\text { Project } \\
\text { ID }\end{array}$ & $\begin{array}{l}\text { Locations } \\
\text { Info }\end{array}$ & $\begin{array}{l}\text { Grid Electricity }{ }^{(1)} \\
\text { (Annual kg CO } \text { eq }_{2} \text { eq }\end{array}$ & $\begin{array}{c}\text { Production }{ }^{(2)} \\
\left.\text { (Annual } \mathrm{kg} \mathrm{CO}_{2} \mathrm{eq}\right)\end{array}$ & $\begin{array}{c}\text { On-site Systems } \\
\left(\text { Life-cycle } \mathrm{kg} \mathrm{CO}_{2} \text { eq }\right)\end{array}$ \\
\hline Nat'l- 098 & Sodus, New York, United States & 171,606 & $(85,607)$ & 36,416 \\
\hline Nat'l- 077 & Honokaa, Hawaii, United States & 428,751 & $(210,154)$ & 64,622 \\
\hline Nat'1-035 & Farragut, Iowa, United States & 504,696 & - & - \\
\hline Nat'l-099 & Phoenix, Arizona, United States & 497,106 & $(182,927)$ & 25,556 \\
\hline Nat'1-075 & Annapolis, California, United States & 262,614 & $(142,127)$ & 36,162 \\
\hline Int'l-056 & Fort McMurray, Alberta, Canada & 577,125 & $(114,094)$ & 17,338 \\
\hline Int'l-106 & Vantaa, Southern Finland, Finland & 314,685 & - & - \\
\hline Int'l-037 & Barreiras, Bahia, Brazil & 2,244 & $(466)$ & 39,814 \\
\hline Int'l-280 & Söke, Aydın, Turkey & 518,189 & $(283,727)$ & 43,486 \\
\hline Int'l-151 & Banjar, Bali, Indonesia & 585,978 & - & - \\
\hline Int'l-214 & Makurdi, Benue, Nigeria & 569,091 & $(216,086)$ & 30,666 \\
\hline Int'l-289 & Belfast, United Kingdom & 401,362 & $(83,252)$ & 18,958 \\
\hline Int'l-192 & Nouakchott, Mauritania & 625,959 & $(294,718)$ & 32,158 \\
\hline Int'1-014 & Gold Coast, Queensland, Australia & 652,659 & $(199,900)$ & 17,760 \\
\hline Int'l-256 & Stellenbosch, South Africa & 5,219 & - & - \\
\hline Int'l-284 & Ajman, United Arab Emirates & 573,376 & $(220,684)$ & 32,024 \\
\hline Int'l-230 & Berdsk, Novosibirskaya, Russia & 363,195 & - & - \\
\hline Int'1-064 & Heze, Shandong, China & 759,588 & $(220,773)$ & 22,126 \\
\hline Int'l-159 & Naples, Campania, Italy & 302,901 & $(168,416)$ & 55,128 \\
\hline Int'l-063 & Coquimbo, Elqui, Chile & 5,721 & $(4,261)$ & 49,732 \\
\hline Int'l-139 & Narasapur, India & 851,427 & $(198,091)$ & 18,892 \\
\hline Int'l-103 & Dessie, Amhara, Ethiopia & 498,533 & $(286,677)$ & 31,040 \\
\hline Int'l-113 & Châteauroux, Centre, France & 14,353 & $(3,631)$ & 20,506 \\
\hline Int'l-167 & Ōita, Japan & 296,800 & $(129,120)$ & 41,832 \\
\hline Int'l-084 & Sabanalarga, Atlántico, Colombia & 658,942 & $(303,417)$ & 43,490 \\
\hline
\end{tabular}

* The data in this table are represented in Figure 4 in the paper

(1) The annual impact from the annual grid electricity consumption.

(2) The annual impacts can be mitigated by using PV and Wind systems on-site.

(3) The impact from the systems on-site comprising the entire system cradle-to-grave life cycle. 